Correspondence

Alasdair M. Cook

alasdair.cook@uni-konstanz.de

\title{
Parvibaculum lavamentivorans gen. nov., sp. nov., a novel heterotroph that initiates catabolism of linear alkylbenzenesulfonate
}

\author{
David Schleheck, ${ }^{1}$ Brian J. Tindall, ${ }^{2}$ Ramón Rosselló-Mora ${ }^{3}$ \\ and Alasdair M. Cook ${ }^{1}$ \\ ${ }^{1}$ Fachbereich Biologie der Universität, D-78457 Konstanz, Germany \\ ${ }^{2}$ DSMZ - Deutsche Sammlung von Mikroorganismen und Zellkulturen GmbH, D-38124 \\ Braunschweig, Germany \\ ${ }^{3}$ CSIC-UIB-Institut Mediterrani d'Estudis Avançats, E-07190 Esporles, Mallorca, Spain
}

\begin{abstract}
Strain DS $-1^{\top}$ is a small $(0.8 \mu \mathrm{m}$ in length and $0.2 \mu \mathrm{m}$ in diameter) heterotrophic bacterium able to $\omega$-oxygenate the commercial surfactant linear alkylbenzenesulfonate (LAS) and shorten the side chain by $\beta$-oxidation to yield sulfophenylcarboxylates. The morphotype is widespread in cultures able to utilize LAS, and a second organism with similar characteristics, strain AN-8, is now available. Utilization of LAS is concomitant with formation of a biofilm, and cells were non-motile. Many surfactants were utilized. The organisms also grew with acetate or octane, but required no biofilm and were motile. Analysis of the gene encoding $16 \mathrm{~S}$ rRNA placed the organisms in the $\alpha$-subclass of the Proteobacteria with a sequence divergence of $>8 \%$ from any species whose name has been validly published. 16S rRNA gene sequence comparison with entries in the GenBank database showed $98 \%$ similarity to an $\alpha$-protobacterial marine isolate, JP57: strain JP57 displayed the same morphotype as strain DS- $1^{\top}$, but it was unable to utilize surfactants or any single source of carbon tested. The lipid components of strains DS $-1^{\top}$ and JP57 were virtually identical. The fatty acids contained ester- and putative amide-linked hydroxy fatty acids, in a combination that is currently unique in the $\alpha$-Proteobacteria. The major respiratory quinone present in both strains was $\mathrm{Q}_{11}$. The polar lipids consisted of phosphatidylglycerol, diphosphatidylglycerol, phosphatidylethanolamine, phosphatidylcholine and two unidentified aminolipids. Data on the $16 \mathrm{~S}$ rRNA gene sequence and the lipid composition indicated that strains DS $-1^{\top}$ and JP57 should be placed in a new genus, for which the name Parvibaculum is proposed. The differences between these strains, supported by DNA hybridizations, lead to the conclusion that strain DS- $1^{\top}$ (=DSM $13023^{\top}=$ NCIMB $\left.13966^{\top}\right)$ is the type strain of a species in the genus Parvibaculum, for which the name Parvibaculum lavamentivorans gen. nov., sp. nov. is proposed.
\end{abstract}

\section{INTRODUCTION}

Surfactants play an important part in our daily lives and our health, be they natural compounds in the lung or gut, naturally derived compounds for cleaning skin (soap), or synthetic compounds for convenient cleaning (e.g. laundry). The major laundry surfactant is linear alkylbenzenesulfonate (LAS), the worldwide production of which is about $2 \cdot 5 \times 10^{6}$ tonnes per annum (Knepper \& Berna, 2003). The complete biodegradation of LAS has been recognized

Published online ahead of print on 20 February 2004 as DOI 10.1099/ ijs.0.03020-0.

Abbreviation: LAS, linear alkylbenzenesulfonate.

The GenBank accession number for the 16S rRNA gene sequence of strain DS- $1^{\top}$ is AY387398. for over 40 years (Sawyer \& Ryckman, 1957), but the first pure culture of a heterotrophic organism (strain DS- $1^{\mathrm{T}}$ ) proven to utilize commercial LAS was not reported until 2000 (Schleheck et al., 2000; see also Hrsák \& Begonja, 1998). Strain DS- $1^{\mathrm{T}}$ catalyses the $\omega$-oxygenation of the LAS side chain and about three rounds of $\beta$-oxidation (Fig. 1); a wide range of products, sulfophenylcarboxylates, sulfophenyldicarboxylates and $\alpha, \beta$-unsaturated sulfophenylcarboxylates, is formed from commercial LAS, which nominally comprises 20 compounds (Dong et al., 2004; Schleheck et al., 2000; see also Eichhorn \& Knepper, 2002). Other organisms degrade the sulfophenylcarboxylates, sulfophenyldicarboxylates and $\alpha, \beta$-unsaturated sulfophenylcarboxylates (Dong et al., 2004; Eichhorn \& Knepper, 2002; Kanz et al., 1998; Schulz et al., 2000; Schleheck et al., 2004). The heterotrophic, LAS-degrading communities 


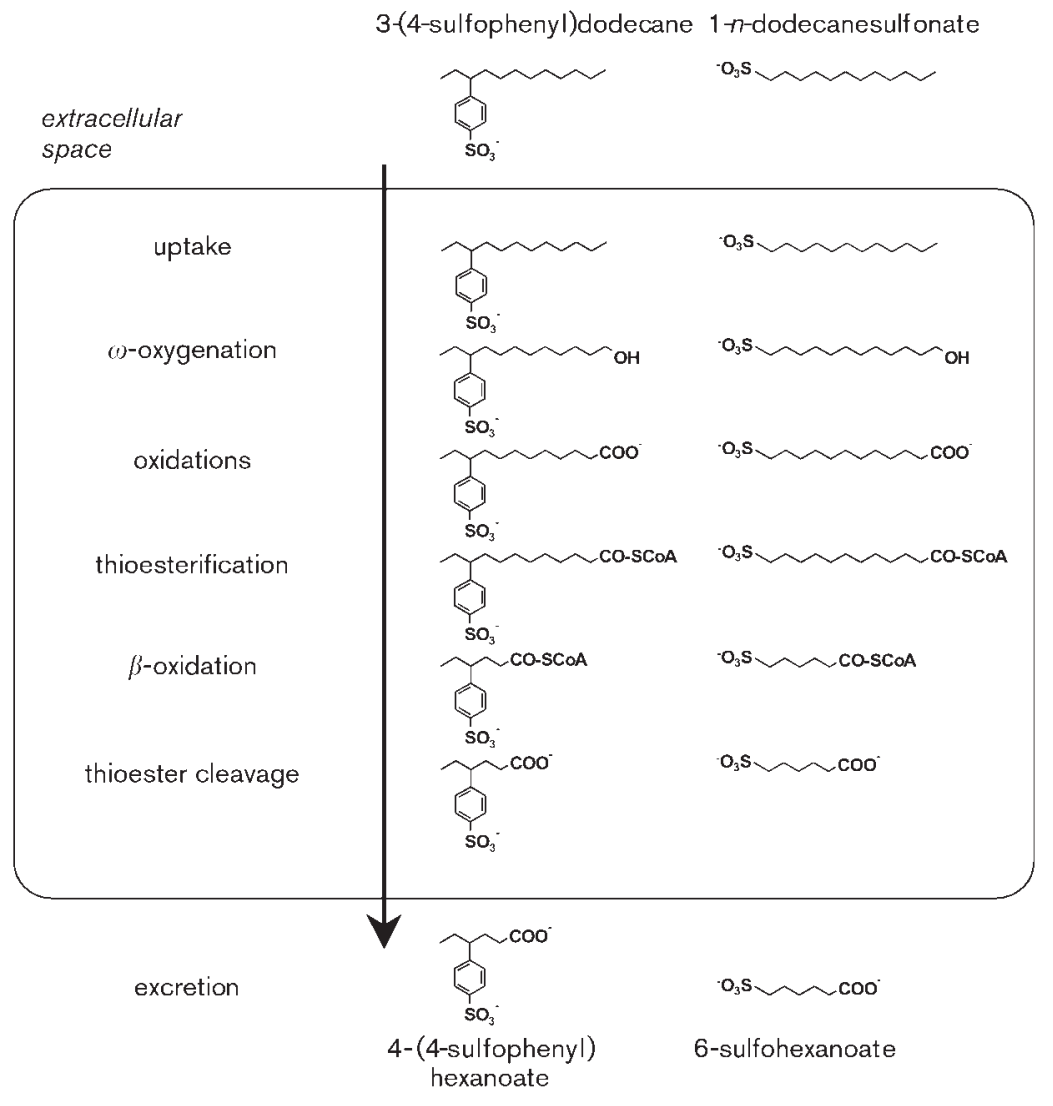

Fig. 1. Degradation of a representative congener of LAS by strain DS-1 ${ }^{\top}$ and the presumed degradation of a primary alkanesulfonate. Strain DS- $1^{\top}$ is apparently the first tier of different microbial communities (Schleheck et al., 2000, 2003) that degrade many different surfactants (see Table 1). from each of six pristine and acclimatized inocula contained organisms morphologically and physiologically similar to strain DS- $1^{\mathrm{T}}$; we were able to isolate one of those six organisms, strain AN-8 (Dong et al., 2004). Other researchers presumably failed to isolate this organism from enrichment cultures, because (i) it grows very slowly on complex medium (where it is rapidly overgrown by other organisms) and is best separated on LAS-salts-agarose (where it can also be easily overgrown), and (ii) it needs a solid support (e.g. glass fibre or polyester fleece) for growth with compounds such as LAS in liquid culture (Schleheck et al., 2000). Strain DS- $1^{\mathrm{T}}$ thus represents the first tier of widespread microbial communities that degrade LAS.

Strain DS- $1^{\mathrm{T}}$ (with strain AN-8) was shown to be a member of the $\alpha$-subclass of the Proteobacteria and was suspected to represent a novel genus (Schleheck et al., 2000). Comparisons with other 16S rRNA gene sequences in databases indicated that the marine $\alpha$-proteobacterium strain JP57 (Eilers et al., 2001) exhibited $98 \%$ sequence identity to strain DS- $1^{\mathrm{T}}$, indicating that a related organism was available for comparison. Four uncultivated members of the $\alpha$-Proteobacteria, from freshwater and marine enrichment cultures that degrade hydrocarbons, also have similar rRNA genes (95-97\% identity to strain JP57) (Chang et al., 2000). It was observed that strains DS- $1^{\mathrm{T}}$ and JP57 do not belong to the same species. We now propose the name Parvibaculum lavamentivorans gen. nov., sp. nov. for strain DS- $1^{\mathrm{T}}$; strain $\mathrm{AN}-8$ is a second representative of Parvibaculum lavamentivorans.

\section{METHODS}

Culture conditions. Strain DS $1^{\mathrm{T}}$ or strain AN 8 was routinely grown in $3 \mathrm{ml}$ mineral salts medium (OECD, 1992) in $30 \mathrm{ml} \mathrm{screw}$ cap tubes to which up to $3 \mathrm{mM}$ LAS (as carbon and energy source) and a solid support were added prior to autoclaving. Three solid supports were used: polyester fleece, an open weave glass fibre and glass particles. Polyester fleece or glass fibre was cut into cubes $\left(1 \mathrm{~cm}^{3}\right)$ and routinely added to $3 \mathrm{ml}$ cultures (Schleheck et al., 2000). Glass particles were generated by macerating glass fibre; they were stored in stock suspensions $\left(10 \mathrm{mg} \mathrm{ml}^{1}\right)$ that were mixed to homogeneity with a magnetically driven stirring bar and portions transferred in wide mouthed pipette tips to growth medium routi nely to $1 \mathrm{mg} \mathrm{ml}{ }^{1}$. [Caution: dry glass particles are a hazard to the eyes and lungs.] Cultures were inoculated $(5 \%, v / v)$ with superna tant fluid from an outgrown LAS culture and incubated on a roller (100 r.p.m.) in the dark at $30^{\circ} \mathrm{C}$. The surfactants in routine use were commercial LAS $\left(\mathrm{C}_{10} \mathrm{C}_{13}\right.$ LAS) (Marlon A350; Hüls) and linear hexadecanediphenyletherdisulfonate (Dowfax 8390; Dow Chemicals). The surfactants listed in Table 1 were tested as sole sources of carbon and energy for growth of strain DS $1^{\mathrm{T}}$. Each com pound was provided at a concentration of $0 \cdot 1 \mathrm{mM}$ (calculated using the mean chain length) in liquid minimal medium containing solid support and inoculated from an outgrown LAS culture (see above); very little growth was possible, but attack on the substrate could be estimated as loss of foaming in the culture (Schleheck et al., 2000). These cultures could be subcultured into medium containing $1 \mathrm{mM}$ surfactant (and solid support) and growth (if any) was reproducible, as was loss of foaming as an indicator of degradation of surfactant. 
Table 1. Growth of strain DS-1 ${ }^{\top}$ in liquid salts medium with various carbon sources, and the effect of a solid support

Four types of surfactant were used: A, anionic; N, non ionic; C, cationic; Z, zwitterionic. NA, Not applicable. Carbon sources that did not support growth with solid support were not tested without solid support.

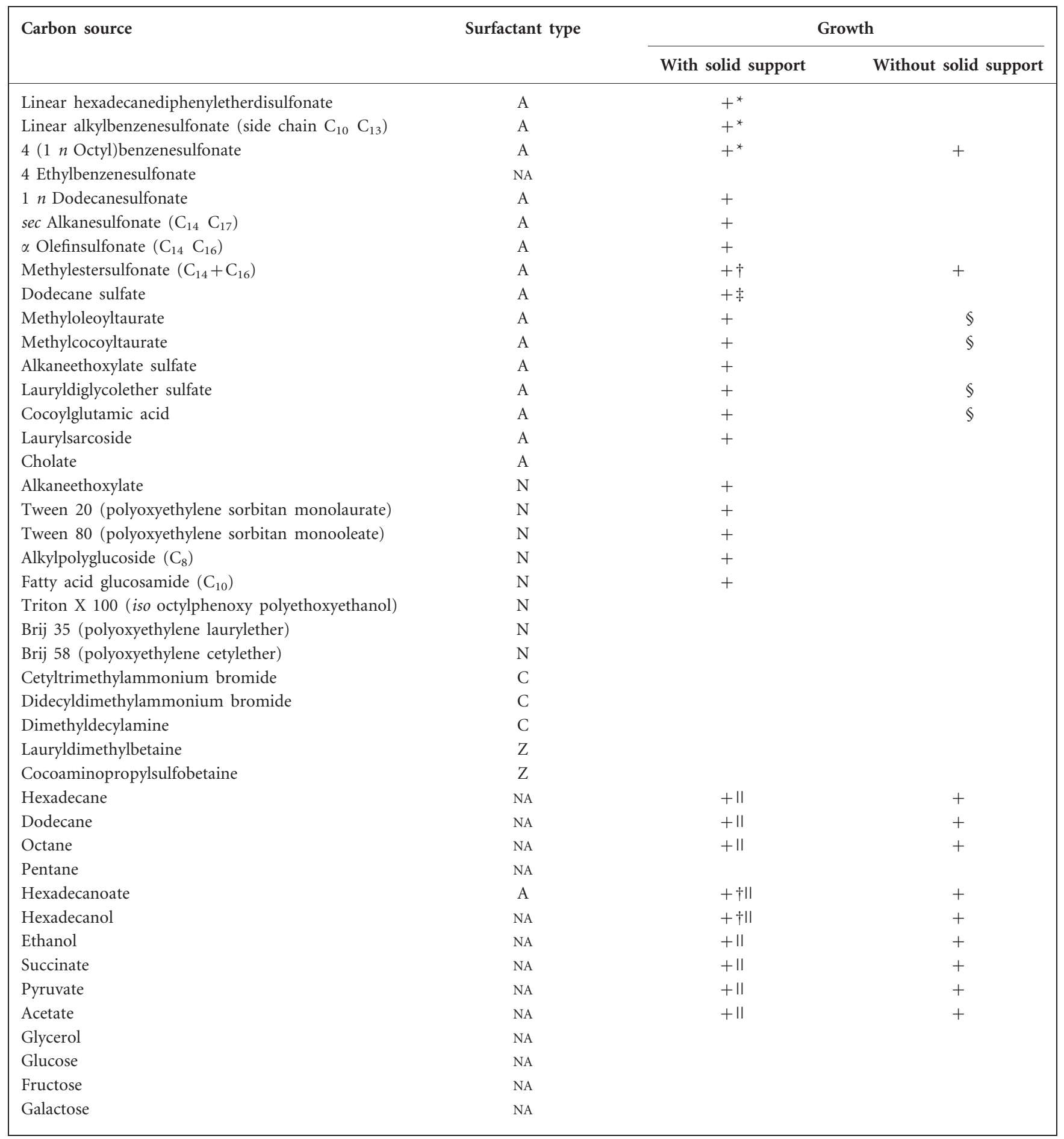

${ }^{*}$ Substrate degradation and product formation were confirmed by HPLC analyses.

$\dagger$ Substrate formed a precipitate in minimal salts medium.

¥ster sulfate recovered quantitatively as inorganic sulfate.

$\S$ Significant growth was independent of a solid support at surfactant concentrations $<1 \mathrm{mM}$.

IIThe organism was motile under these conditions. 
These cultures were also streaked on agar salts medium which con tained the appropriate surfactant. Growth with more usual (non surface active) laboratory sources of carbon and energy did not need to be acclimatized to the substrate to yield reproducible results. Volatile alkanes for plate cultures were provided in the gas phase. The identity of the organism subsequent to tests for substrate range was checked by plating on Luria Bertani medium, on plates with $1 \mathrm{mM}$ LAS salts medium and by behaviour in $1 \mathrm{mM}$ LAS salts medium with and without a solid phase. Pentane, octane, dodecane, hexadecane or octanol ( $1 \mu \mathrm{l}$ by syringe) was added directly to $3 \mathrm{ml}$ liquid salts medium in a screw capped tube. Hexadecanol or sodium hexadecanoate was provided as a suspension of particles in liquid culture medium.

Strain JP57, kindly provided by J. Peplies and R. Amann (Max Planck Institute for Marine Microbiology, Bremen), was routinely grown in a synthetic sea water salts medium, MPM m (Eilers et al., 2001), with peptone $\left(10 \mathrm{~g} \mathrm{l}^{1}\right)$ as the carbon source, or in peptone medium $(10 \mathrm{~g}$ peptone, $5 \mathrm{~g} \mathrm{NaCl}$ and $0 \cdot 1 \mathrm{~g} \mathrm{CaCl}_{2} \cdot 2 \mathrm{H}_{2} \mathrm{O}^{1}$ ) in which strain DS $1^{\mathrm{T}}$ also grew (cf. DSM medium 884). The substrate range of strain JP57 was tested in MPM m medium, in MPM m medium which was supplemented with $6 \mathrm{mM} \mathrm{NH}_{4} \mathrm{Cl}$ and $0.25 \mathrm{mM} \mathrm{K}_{2} \mathrm{HPO}_{4}$ and in mineral salts medium (see above) to which peptone or a defined carbon source (see Table 1) had been added. The seven vitamins solution (cf. DSM medium 503) and trace elements solution (cf. DSM medium 320) are described elsewhere (Denger et al., 1999).

Morphology, physiology and biofilm staining. Culture purity, cell morphology, motility and spore formation were examined microscopically. The Gram reaction was assayed using the $\mathrm{KOH}$ test (Gregersen, 1978). Oxidase and catalase tests were carried out using standard methods (Gerhardt et al., 1994). Strain DS $1^{\mathrm{T}}$ grew in a biofilm on the polyester fleece when utilizing LAS. The biofilm on a section of fleece from $0.5 \mathrm{mM}$ LAS salts medium was visualized in situ by staining with ruthenium red $\left(2 \cdot 5 \mu \mathrm{g} \mathrm{ml}{ }^{1}\right)$, whereas the cells were stained with $4^{\prime}, 6$ diamidino 2 phenylindole $\left(1 \mu \mathrm{g} \mathrm{ml}{ }^{1}\right)$.

Analytical methods. Respiratory lipoquinones and polar lipids were extracted from freeze dried cell material $(100 \mathrm{mg})$ using a two stage method (Tindall, 1990a, b). The lipoquinones were separated by TLC, and UV absorbing bands corresponding to menaquinones or ubiquinones were removed from the plate and further ana lysed by reversed phase HPLC. Polar lipids were separated by two dimensional, silica gel TLC; total lipid material and specific functional groups were detected using dodecamolybdophosphoric acid (total lipids), Zinzadze reagent (phosphate), ninhydrin (free amino groups), periodate Schiff ( $\alpha$ glycols), Dragendorff (quatern ary nitrogen) and anisaldehyde sulfuric acid (glycolipids) as described previously (Tindall, 1990a, b). Fatty acids were analysed as the methyl ester derivatives prepared from $10 \mathrm{mg}$ dry cell material. Cells were subjected to differential hydrolysis to detect ester linked and non ester linked (amide bound) fatty acids (Labrenz et al., 1998). Fatty acid methyl esters were analysed by GC using a $0 \cdot 2 \mu \mathrm{m} \times 25 \mathrm{~m}$ non polar capillary column and flame ionization detection. The run conditions were as follows: injection and detector port temperature, $300^{\circ} \mathrm{C}$; inlet pressure, $60 \mathrm{kPa}$; split ratio, $50: 1$; injection volume, $1 \mu \mathrm{l}$; temperature program, $130310^{\circ} \mathrm{C}$ at a rate of $4{ }^{\circ} \mathrm{C}$ min ${ }^{1}$. The identity of each fatty acid was confirmed by GC MS, using conditions described in Strömpl et al. (1999). Hydroxy fatty acids were confirmed by either the presence of a major peak at $\mathrm{m} / z 103$ (3 OH fatty acids) or the loss of 59 mass units from the mole peak (i.e. $\mathrm{m} / \mathrm{zM}^{+}$59, for $2 \mathrm{OH}$ fatty acids). The position of double bonds was confirmed using dimethysulfide adducts (Nichols et al., 1986).

LAS and sulfophenylcarboxylates were determined by HPLC
(Schleheck et al., 2000). Sulfate was quantified turbidimetrically (Sörbo, 1987). Protein solubilized from whole cells was quantified in a Lowry type reaction (Kennedy \& Fewson, 1968).

Extraction of genomic DNA, PCR mediated amplification of the 16S rRNA gene, purification of the PCR products and sequence analysis were done as described elsewhere (Rainey et al., 1996). Sequence reactions were analysed using the Applied Biosystems 373A DNA sequencer. The sequence was aligned manually to $16 \mathrm{~S}$ rRNA gene sequences of representative micro organisms belonging to the domain Bacteria by using the alignment editor ae2 (Maidak et al., 1996). A phylogenetic tree was generated using the algorithm of De Soete (1983) (carried out by the DSMZ). DNA DNA hybridizations and DNA G $+\mathrm{C}$ content were determined as described previously (Ziemke et al., 1998).

\section{RESULTS AND DISCUSSION}

\section{Morphology and growth range}

Strain DS- $1^{\mathrm{T}}$ was found to be an aerobic, uniform, nonmotile, Gram-negative, short rod $(0 \cdot 8 \mu \mathrm{m}$ in length and $0 \cdot 2 \mu \mathrm{m}$ in diameter) when growing with commercial LAS in suspended culture, associated with a biofilm (see also below) of the same organism on a solid support. The requirement for a solid support for growth with LAS is illustrated in Fig. 2(a, b). Non-inoculated medium foamed on shaking, and turbidity due to glass particles was visible (Fig. 2a, tube 1). The inoculum did not grow after 8 days in the absence of glass particles (Fig. 2a, tube 2), but in the presence of particles strain DS- $1^{\mathrm{T}}$ degraded LAS (no foam on shaking) and caused the particles to clump due to the formation of biofilm (see sediment) (Fig. 2a, tube 3). When a growth curve was generated (Fig. 2b), no growth was detected after 8 days without a support, but the addition of a small number of glass particles (or polyester fleece, not shown) allowed growth; more glass particles allowed growth with a shorter lag phase. The organism did not grow in the presence of glass particles without LAS (not shown). Sorptive properties of the solid support were irrelevant for growth, since glass particles were non-sorptive, whereas polyester fleece could bind up to $50 \%$ of the LAS (Fig. 2c), though long-chain congeners were more extensively bound. Strain DS- $1^{\mathrm{T}}$ showed no growth in the absence of glass particles (or fleece), as illustrated in Fig. 2(a, b), but prolonged incubation could lead to degradation of some LAS (not shown). The incubation time before degradation occurred could be extended by filtering the medium $(0 \cdot 2 \mu \mathrm{m}$ pore size), so we presume that many types of particle can serve as a solid support for the development of a biofilm.

Strain DS- $1^{\mathrm{T}}$ was found to be able to metabolize a wide range of anionic and non-ionic surfactants (Table 1). In almost every case, a solid support was essential for growth. There were two apparent exceptions (Table 1): methylestersulfonate, which precipitated in the salts medium and thus supplied its own solid support, and octylbenzenesulfonate, for which we have no explanation. The only anionic surfactant (cholate) that did not support growth contained no hydrocarbon chain. The non-ionic surfactants that did 
(a)

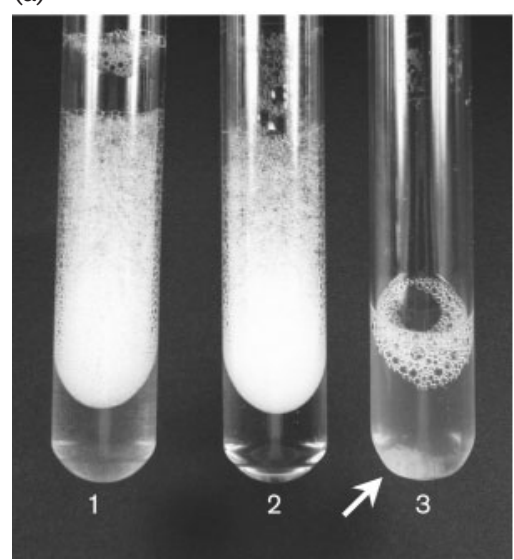

(b)

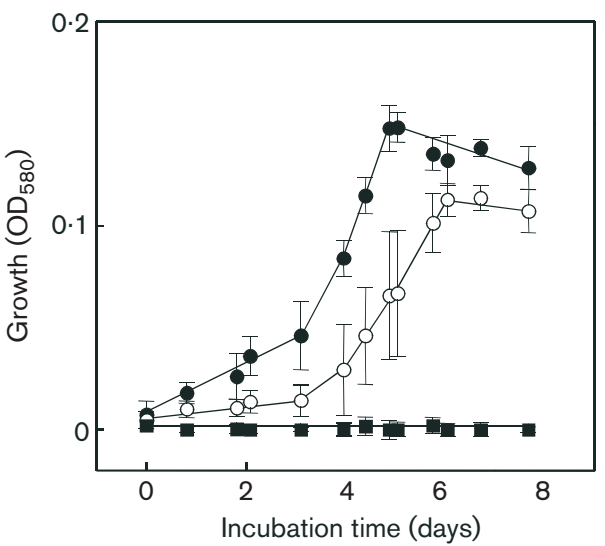

(c)

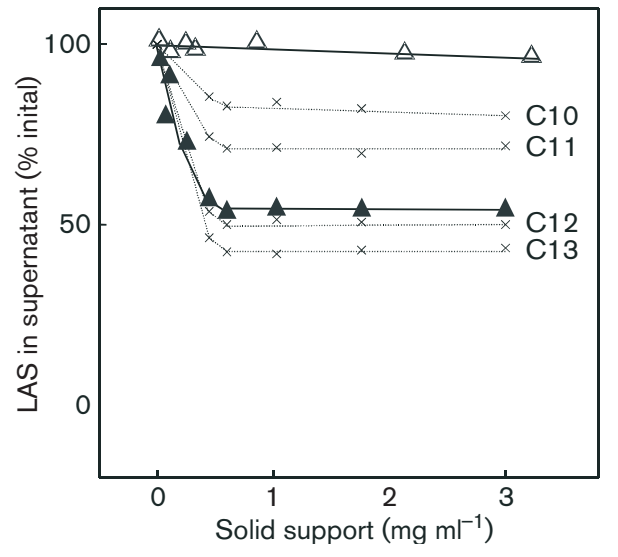

Fig. 2. Growth of strain DS-1 ${ }^{\top}$ in LAS-salts medium as a function of the presence of solid support, assayed as the disappearance of substrate (a) or as growth (b), and the sorbtion of LAS to different supports (c). Cultures of strain DS- $1^{\top}$ were incubated for 8 days $(a, b)$. (a) The spot test (shaking) indicated the presence of surfactant as foam: tube 1, noninoculated control with glass particles $\left(1 \mathrm{mg} \mathrm{ml}^{1}\right)$; tube 2, inoculated culture in the absence of glass particles; tube 3, inoculated culture in the presence of glass particles $\left(1 \mathrm{mg} \mathrm{ml}^{1}\right)$; arrow, indicates the sediment of clumped glass particles. (b) Growth could be assayed as turbidity $\left(\mathrm{OD}_{580}\right.$; see Methods). The values are the means of three independent experiments and the error bars show the standard deviation: $\mathbf{\square}$, no glass particles; $\bigcirc, 0.5 \mathrm{mg}$ glass particles $\mathrm{ml}{ }^{1} ; \boldsymbol{\bullet}, 1 \mathrm{mg}$ glass particles $\mathrm{ml}^{1}$. (c) Surfactants can be difficult to quantify in growth medium, as shown: a fixed concentration of LAS in sterile salts medium was incubated with different amounts of solid support (polyester fibre or glass particles), then the LAS concentration in solution was determined after $12 \mathrm{~h}$ (HPLC). Values of the negative controls (no solid support) were set as $100 \%$. LAS concentration (all congeners) in solution (solid lines) is shown in the presence of polyester fleece $(\boldsymbol{\Delta})$ or glass fibre $(\triangle)$ ). Detailed analysis of HPLC chromatograms showed that a larger proportion of the longer-chain congeners (e.g. C13) of LAS sorbed to the fleece, whereas there was no sorption to the glass particles. LAS desorbs from the fleece during growth: the same concentration of sulfophenylcarboxylate is observed at the end of growth in the presence of glass particles or fleece (not shown).

not support growth contain a branched hydrocarbon chain (Triton; Fluka) or long polyethoxyethylene chains (Brij; Aldrich). Cationic surfactants were not utilized (Table 1), even when silica gel was added to reduce toxicity (see van Ginkel et al., 1992). Zwitterionic compounds were also not utilized (Table 1). Thus strain DS- ${ }^{\mathrm{T}}$ utilizes all representatives of the two major classes of surfactants used in laundry products in Germany, anionics and non-ionics.

In contrast to the generality of needing a solid support for growth with surfactants, strain DS- ${ }^{\mathrm{T}}$ utilized some alkanes, alkanoates, alcohols and short-chain acids (e.g. acetate), without the requirement for a solid support (Table 1). Under these latter conditions, the organism grew in suspension (Fig. 3a) and was motile. Strain DS- $1^{\mathrm{T}}$ grew slowly in complex medium without a solid support, e.g. in peptone-salts medium ( 5 days): the organism was motile and was found as single cells or in short chains (two to five organisms). The sugars tested were not utilized (Table 1).

Quantification of molar growth yields indicated that strain DS- $1^{\mathrm{T}}$ usually degraded alkyl chains of surfactants by excision of about six carbon atoms, presumably until $\beta$-oxidation was hindered by a (polar) substituent, as indicated in Fig. 1 for the utilization of both LAS and dodecanesulfonate. No sulfate was recovered from any sulfonated surfactant. Strain DS- $1^{\mathrm{T}}$ grew poorly with the alkylpolyglucoside and with the fatty acid glucosamide (Table 1), but did reduce the foaming, so presumably the short alkyl chain offered only about one round of $\beta$ oxidation for growth. In contrast, the growth yield with dodecyl sulfate indicated complete utilization and, correspondingly, sulfate was recovered at high yields.

Growth of strain AN-8 with LAS also showed an absolute requirement for a solid support, concomitant with formation of a biofilm and the presence of non-motile cells. Growth with e.g. acetate or octane involved motile cells; no solid support was necessary and no biofilm was formed. Growth in peptone-salts medium involved single cells and short chains. Sugars did not support growth. The cell morphology was indistinguishable from that of strain DS- $1^{\mathrm{T}}$.

Marine isolate strain JP57 grew on synthetic-sea-water agar plates without an additional carbon source in pinpoint colonies, as described in the original publication (Eilers et al., 2001). The organism grew in peptone medium after 5 days incubation and was morphologically indistinguishable from strains DS- $1^{\mathrm{T}}$ and AN-8. Strain JP57 failed to grow 

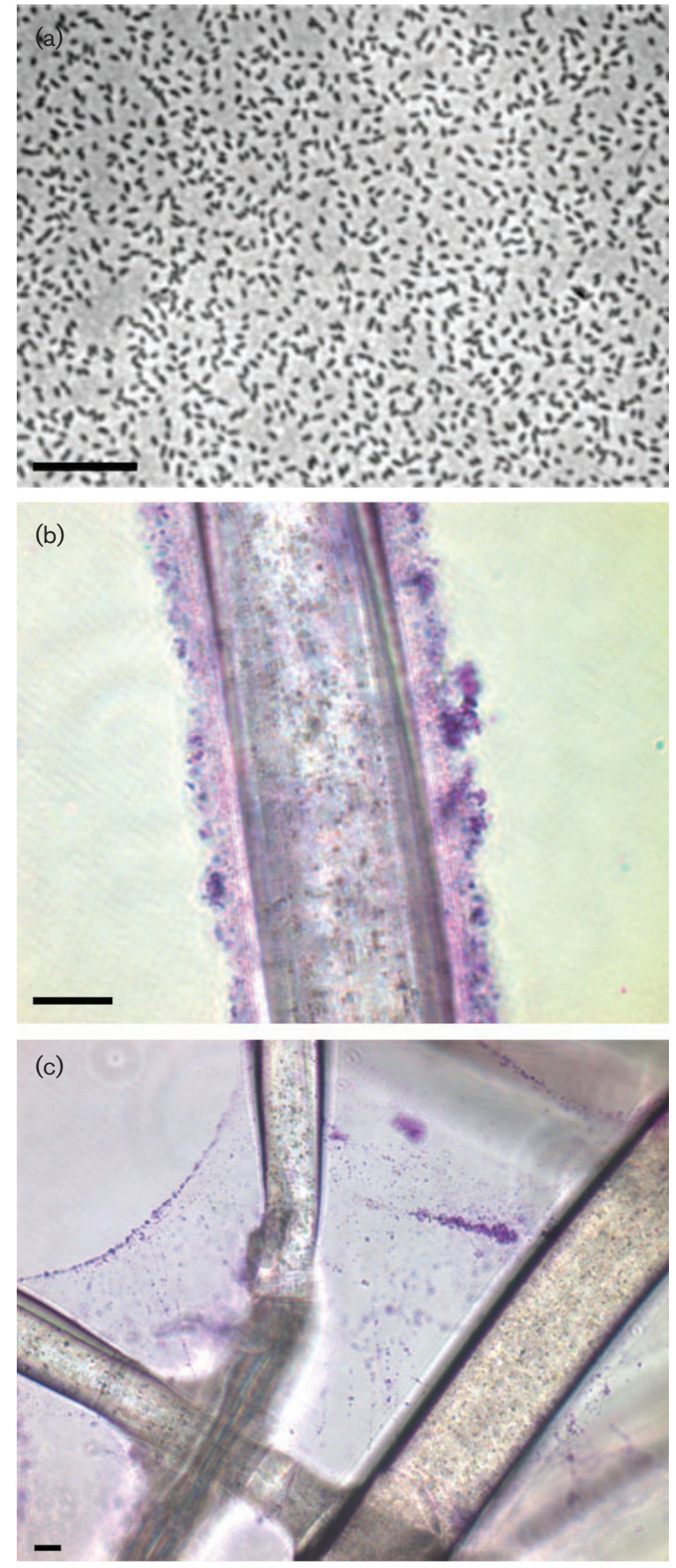

Fig. 3. Photomicrographs of phase-contrast microscopy of strain $D S-1^{\top}$ during growth in acetate-salts medium (a) or during growth in LAS-salts medium as biofilm on polyester fleece (b, sheath; c, gossamer form) (stained with ruthenium red). Bars, $10 \mu \mathrm{m}$.

with LAS, sodium dodecyl sulfate, methylestersulfonate, octane, dodecane, hexadecane, hexadecanol, hexadecanoate, acetate, succinate, pyruvate, ethanol or sugars as carbon source, either when supplied in minimal-salts medium or when supplied in supplemented artificial sea-salts medium. We found no single source of carbon for the organism.
Neither the addition of solid support nor a vitamin supplement had any effect.

\section{Formation of biofilm}

The first visible indication of growth of strain DS- $1^{\mathrm{T}}$ in LASsalts medium was the formation of biofilm on the solid support; later during growth, bacteria were also found in suspension. Two forms of biofilm could be observed on polyester fibres (Fig. 3b, c). The first form was a densely packed sheath of cells around a fibre (Fig. 3b). Fig. 3(b) was generated with ruthenium red as the stain, but $4^{\prime}, 6$ diamidino-2-phenylindole, methylene blue or Congo red gave similar results (not shown). The second one was a gossamer form (Fig. 3c), usually found linking two or more fibres. Each form could be removed from the support by, for example, vortexing and was of a soft consistency. The gossamer form only was observed on glass fibres (not shown) and negligible amounts of biofilm (gossamer) were observed on glass particles, e.g. in the sediment from the culture fluid: the bacteria grew predominantly in suspension and were non-motile. We assume that the biofilm observed on glass particles was continuously disrupted during incubation on a roller.

Growth in acetate-salts medium involved motile cells in suspension (see above). When LAS was added to an acetatesalts culture, a solid support was needed for growth; a biofilm was formed and non-motile cells were observed in the presence of LAS.

Some growth substrates were precipitated in the medium. In the case of methylestersulfonate surfactant (Table 1), a biofilm could be detected on the particles by using staining (ruthenium red, not shown) and only non-motile cells were observed in the medium. In contrast, growth with the insoluble substrates hexadecanoate or hexadecanol did not involve a biofilm (ruthenium red) and cells in the culture were motile. We presume that the formation of biofilm with strains DS- $1^{\mathrm{T}}$ and $\mathrm{AN}-8$, and the switch from motile to nonmotile, are protective responses to the risks inherent in growing with membrane-solubilizing agents.

\section{5 rDNA sequence analysis, chemotaxonomy and DNA-DNA hybridization}

Published data on the sequences of $16 \mathrm{~S}$ rRNA genes place strains DS- $1^{\mathrm{T}}(1451 \mathrm{nt})$ and JP57 (1356 nt), which share $98 \%$ similarity, in the $\alpha$-Proteobacteria, where the nearest, well-described organism is Rhodobium (formerly Rhodobacter) marinum, which shares only $92 \%$ similarity (Eilers et al., 2001; Schleheck et al., 2000). The morphological similarities of these two organisms support the implication from the sequence that they are closely related, but differences (e.g. substrate range) are also apparent. A $400 \mathrm{bp}$ sequence of the 16S rRNA gene from strain AN-8 was identical to the corresponding sequence of strain DS- $1^{\mathrm{T}}$; this, together with the essentially identical morphology and 
physiology, supports the hypothesis that they are members of one species.

Examination of the respiratory lipoquinone composition of both strain JP57 and strain DS- $1^{\mathrm{T}}$ showed that ubiquinones are the sole respiratory quinones present. Furthermore, the major lipoquinone had 11 isoprenologues in the side chain, i.e. $\mathrm{Q}_{11}$.

The fatty acids comprised both saturated and unsaturated straight-chain fatty acids, as well as hydroxylated fatty acids, strains DS- $1^{\mathrm{T}}$ and JP57 showing only quantitative differences (Table 2). Differential hydrolysis of the cells indicated that some of the hydroxy fatty acids were probably amide-linked (Table 2). The polar lipids comprised the phospholipids phosphatidylglycerol, diphosphatidylglycerol, phosphatidylethanolamine and phosphatidylcholine (Fig. 4). In addition, two aminolipids were present which were detected between phosphatidyl glycerol and phosphatidyl choline (Fig. 4). There were quantitative differences in the ratio of these two lipids, with one predominating in strain JP57. This causes the lower lipid to be partially obscured by the upper lipid, but this was simply a 'crowding effect' on the TLC plate.

The DNA G + C contents of strains DS- $1^{\mathrm{T}}$ and JP57 were determined to be $64 \cdot 5( \pm 1) \mathrm{mol} \%$ and $63 \cdot 7( \pm 1 \cdot 3) \mathrm{mol} \%$, respectively.

The use of a combination of the 16S rRNA sequence data and the lipid composition provides a convenient way of quickly determining the differences from, and/or similarities to, other organisms. The presence of ubiquinones as the sole respiratory quinones is indicative of the fact that strains DS$1^{\mathrm{T}}$ and JP57 are members of the $\alpha$-, $\beta$ - or $\gamma$-subclass of the Proteobacteria. The presence of $\mathrm{Q}_{11}$ is distinctive in that the

Table 2. Percentage composition of the fatty acids present in strains DS-1 ${ }^{\top}$ and JP57

Methods 1 and 2 (Labrenz et al., 1998) released ester linked and ester linked plus amide linked fatty acids, respectively. tr, Trace amounts.

\begin{tabular}{|lccccc|}
\hline \multirow{2}{*}{ Fatty acid } & \multicolumn{2}{c}{ Strain DS $\mathbf{1}^{\mathrm{T}}$} & & \multicolumn{2}{c|}{ Strain JP57 } \\
\cline { 2 - 3 } \cline { 5 - 6 } & Method 1 & Method 2 & & Method 1 & Method 2 \\
\hline 3 OH 14:0 & $6 \cdot 3$ & $5 \cdot 7$ & & $5 \cdot 3$ & $5 \cdot 5$ \\
$16: 0$ & $6 \cdot 5$ & $6 \cdot 1$ & & $2 \cdot 7$ & $2 \cdot 4$ \\
3 OH 16:1 & $1 \cdot 4$ & $1 \cdot 3$ & & $\operatorname{tr}$ & $\operatorname{tr}$ \\
3 OH 16:0* & $1 \cdot 2$ & $9 \cdot 1$ & & $\operatorname{tr}$ & $7 \cdot 6$ \\
$18: 1 \omega 7 c$ & $50 \cdot 4$ & $47 \cdot 4$ & & $72 \cdot 1$ & $66 \cdot 4$ \\
$18: 0$ & $9 \cdot 7$ & $9 \cdot 0$ & & $3 \cdot 4$ & $3 \cdot 2$ \\
Unknown & $\operatorname{tr}$ & $\operatorname{tr}$ & & $0 \cdot 9$ & $1 \cdot 2$ \\
cyclo 19:0 & $13 \cdot 6$ & $11 \cdot 8$ & & $6 \cdot 7$ & $5 \cdot 3$ \\
2 OH 18:1 & $2 \cdot 8$ & $2 \cdot 6$ & & $2 \cdot 5$ & $2 \cdot 5$ \\
cyclo 2 OH 19:0 & $8 \cdot 1$ & $7 \cdot 0$ & & $6 \cdot 4$ & $5 \cdot 9$ \\
\hline
\end{tabular}

${ }^{\star}$ Putative amide linked fatty acids.
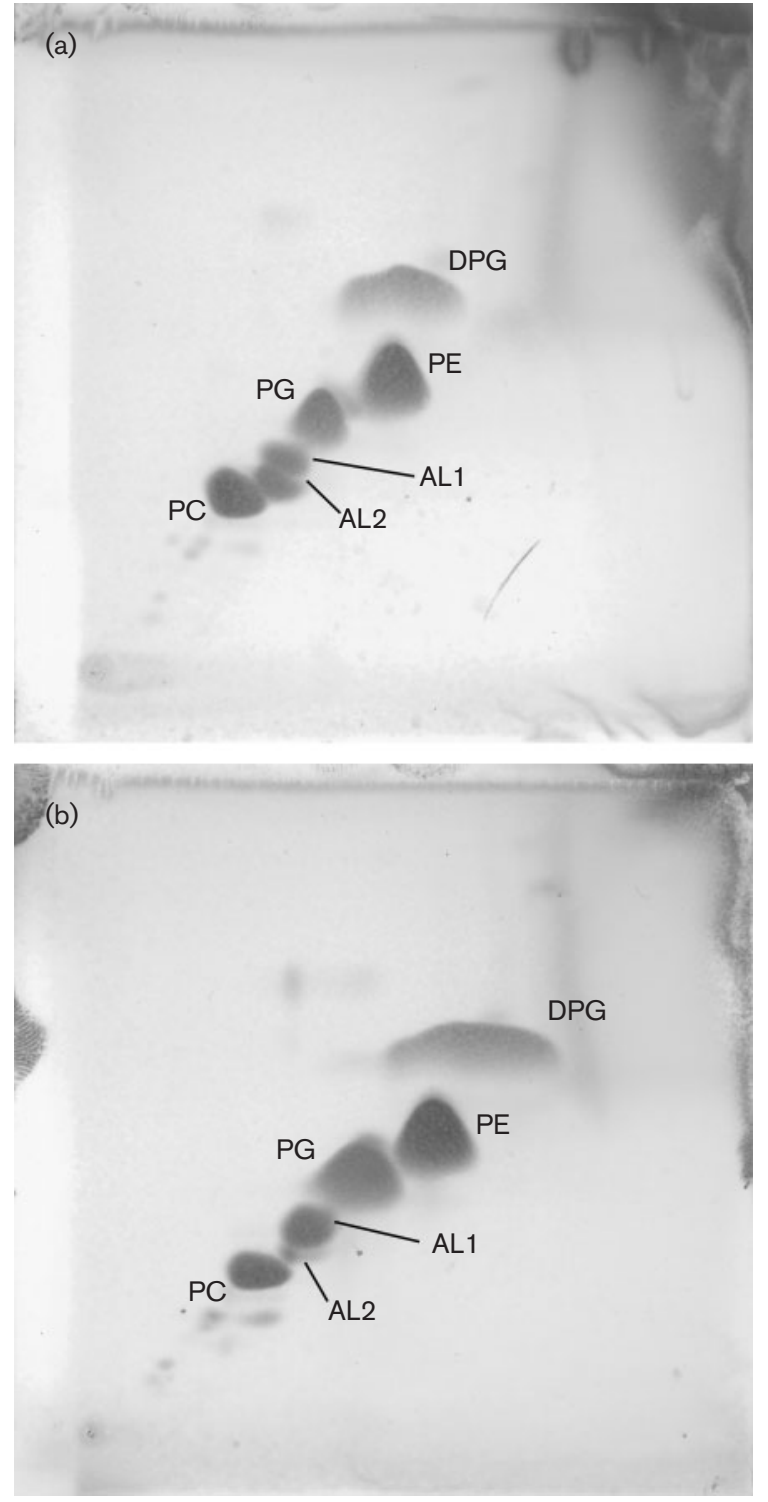

Fig. 4. Two-dimensional thin-layer chromatograms of the polar lipids of strain DS-1 ${ }^{\top}$ (a) and JP57 (b) stained with $5 \%$ ethanolic molybdophosphoric acid. Solvents: first dimension, chloroform/methanol/water (65:25:4, by vol.); second dimension, chloroform/methanol/acetic acid/water (80:12:15:4, by vol.). DPG, diphosphatidylglycerol; PE, phosphatidylethanolamine; PG, phosphatidylglycerol; PC, phosphatidylcholine; AL1 and AL2, aminolipids of unknown structure.

presence of this compound as the sole major quinone has previously been found only in members of the genus Hyphomonas (Sittig \& Hirsch, 1992; Urakami \& Komagata, 1987), in the $\alpha$-subclass of the Proteobacteria. $\mathrm{Q}_{11}$ is also known to occur in members of the genus Legionella (a member of the $\gamma$-subclass of the Proteobacteria), but it is usually accompanied by additional isoprenologues (see Collins \& Gilbart, 1983). The fatty acid patterns of the two organisms, showing large amounts of $18: 1 \omega 7 c$ (Table 2), are not atypical of members of the $\alpha$-subclass of the 
Proteobacteria. However, the distribution of the hydroxy fatty acids does appear to be a unique feature of these two organisms. In particular, the fact that some of the hydroxy fatty acids are ester-linked, while others are presumably amide-linked, allows further differentiation of these two strains from other members of the $\alpha$-subclass of the Proteobacteria. The presence of the phospholipids phosphatidylglycerol, diphosphatidylglycerol, phosphatidylethanolamine and phosphatidylcholine is also characteristic, together with the respiratory lipoquinone and fatty acid data, of certain subgroups within the $\alpha$-subclass of the Proteobacteria. However, the presence of the two additional aminolipids appears to be distinctive for these two organisms.

DNA-DNA hybridization with strains DS- ${ }^{\mathrm{T}}$ and JP57 showed $42 \%$ binding, which is similar to the value obtained against a strain of Pseudomonas putida (35\%), whereas the positive control was $100 \%$. We thus conclude that strains DS- $1^{\mathrm{T}}$ and JP57 represent different strains of the same higher taxon within the $\alpha$-subclass of the Proteobacteria. In the present work, we have concentrated on the taxonomic position of strain DS- $1^{\mathrm{T}}$; a taxonomic treatment of strain JP57 may appear at a later date.

\section{Description of Parvibaculum lavamentivorans gen. nov., sp. nov.}

Parvibaculum lavamentivorans [Par.vi.ba'cu.lum. L. adj. parvus small; L. neut. n. baculum stick; N.L. neut. n. Parvibaculum small stick. la.va.men.ti.vo'rans. L. v. lavo to wash, L. neut. n. suffix - mentum agent of (specified) action; L. v. voro to consume; N.L. neut. part. adj. lavamentivorans consuming (chemicals) used for washing].

Cells are aerobic, uniform, Gram-negative, short rods $(0.8 \mu \mathrm{m}$ in length and $0.2 \mu \mathrm{m}$ in diameter). Oxidase- and catalase-positive. Mesophilic. Motile when growing with acetate, octane or in complex medium. $\mathrm{Q}_{11}$ is the major respiratory quinone. Straight-chain saturated and unsaturated, as well as ester- and (presumptively) amide-linked hydroxy-fatty acids are present in membrane fractions (see Table 2). The major polar lipids are phosphatidylglycerol, diphosphatidylglycerol, phosphatidylethanolamine, phosphatidylcholine and two unidentified aminolipids. $16 \mathrm{~S}$ rRNA gene sequence analysis indicates that this taxon is a member of the $\alpha$-subclass of the Proteobacteria, with $>8 \%$ sequence divergence with respect to any other species, within this subclass, whose name has been validly published. The $\mathrm{G}+\mathrm{C}$ content is $64 \mathrm{~mol} \%$. Strain DS- $\mathrm{1}^{\mathrm{T}}$ grows with LAS and other surfactants via $\omega$-oxygenation and $\beta$-oxidation of the alkyl chain to produce short-chain sulfophenylcarboxylates. Growth in surfactant medium involves biofilm formation on solid support (polyester fleece, glass particles) and non-motile cells.

The type species of the genus is Parvibaculum lavamentivorans. Strain DS- $1^{\mathrm{T}}\left(=\mathrm{DSM} 13023^{\mathrm{T}}=\mathrm{NCIMB} 13966^{\mathrm{T}}\right)$ is the type strain of Parvibaculum lavamentivorans.

\section{ACKNOWLEDGEMENTS}

We are grateful to J. Peplies and R. Amann, who kindly made strain JP57 available to us, and to B. Schink for advice on Greek, Latin and nomenclature. L. Cavalli (Condea Augusta), P. Klug (Clariant), T. P. Knepper (ESWE), J. Steber (Henkel) and C. G. van Ginkel (Akzo Nobel) generously supplied surfactants. Funding was made available by the European Union (SUITE: ENV4 CT98 0723), the Stiftung Umwelt und Wohnen and the University of Konstanz.

\section{REFERENCES}

Chang, Y. J., Stephen, J. R., Richter, A. P. \& 7 other authors (2000). Phylogenetic analysis of aerobic freshwater and marine enrichment cultures efficient in hydrocarbon degradation: effect of profiling method. J Microbiol Methods 40, 1931.

Collins, M. D. \& Gilbart, J. (1983). New members of the coenzyme Q series from the Legionellaceae. FEMS Microbiol Lett 16, 251255.

Denger, K., Stackebrandt, E. \& Cook, A. M. (1999). Desulfonispora thiosulfatigenes gen. nov., sp. nov., a widespread, taurine fermenting, thiosulfate producing, anaerobic bacterium. Int J Syst Bacteriol 49, 15991603.

De Soete, G. (1983). On the construction of "optimal" phylogenetic trees. Z Naturforsch [C] 38, 156158.

Dong, W., Eichhorn, P., Radajewski, S., Schleheck, D., Denger, K., Knepper, T. P., Murrell, J. C. \& Cook, A. M. (2004). Parvibaculum lavamentivorans converts linear alkylbenzenesulfonate (LAS) surfactant to sulfophenylcarboxylates, $\alpha, \beta$ unsaturated sulfophenyl carboxylates and sulfophenyldicarboxylates, which are degraded in communities. J Appl Microbiol 96, 630640.

Eichhorn, P. \& Knepper, T. P. (2002). $\alpha, \beta$ Unsaturated sulfophe nylcarboxylate intermediates detected during aerobic degradation of linear alkylbenzenesulfonate (LAS) surfactant: direct evidence for $\omega$ oxygenation followed by $\beta$ oxidations by liquid chromatography mass spectrometry. Environ Toxicol Chem 21, 18.

Eilers, H., Pernthaler, J., Peplies, J., Glöckner, F. O., Gerdts, G. \& Amann, R. (2001). Isolation of novel pelagic bacteria from the German Bight and their seasonal contributions to surface pico plankton. Appl Environ Microbiol 67, 51345142.

Gerhardt, P., Murray, R. G. E., Wood, W. A. \& Krieg, N. R. (1994), Methods for General and Molecular Bacteriology. Washington, DC: American Society for Microbiology.

Gregersen, T. (1978). Rapid method for distinction of Gram negative from Gram positive bacteria. Eur J Appl Microbiol Biotechnol 5, 123127.

Hrsák, D. \& Begonja, A. (1998). Growth characteristics and metabolic activities of the methanotrophic heterotrophic ground water community. J Appl Microbiol 85, 448456.

Kanz, C., Nölke, M., Fleischmann, T., Kohler, H.-P. E. \& Giger, W. (1998). Separation of chiral biodegradation intermediates of linear alkylbenzenesulfonates by capillary electrophoresis. Anal Chem 70, 913917.

Kennedy, S. I. T. \& Fewson, C. A. (1968). Enzymes of the mandelate pathway in bacterium N.C.I.B. 8250. Biochem J 107, 497506.

Knepper, T. P. \& Berna, J. L. (2003). Surfactants: properties, production, and environmental aspects. In Analysis and Fate of Surfactants in the Aquatic Environment, pp. 1 50. Edited by T. P. Knepper, D. Barceló \& P. de Voogt. Amsterdam: Elsevier.

Labrenz, M., Collins, M. D., Lawson, P. A., Tindall, B. J., Braker, G. \& Hirsch, P. (1998). Antarctobacter heliothermus gen. nov., sp. nov., a budding bacterium from hypersaline and heliothermal Ekho Lake. Int J Syst Bacteriol 48, 13631372. 
Maidak, B. L., Olsen, G. J., Larsen, N., Overbeek, R., McCaughey, M. J. \& Woese, C. R. (1996). The ribosomal database project (RDP). Nucleic Acids Res 24, 8285.

Nichols, P. D., Guckert, J. B. \& White, D. C. (1986). Determination of monounsaturated fatty acid double bond position and geometry for microbial monocultures and complex consortia by capillary GC MS of their dimethyl disulphide adducts. J Microbiol Methods 5, 4955.

OECD (1992). Guidelines for Testing Chemicals. Paris: Organization for Economic Cooperation and Development.

Rainey, F. A., Ward-Rainey, N., Kroppenstedt, R. M. \& Stackebrandt, E. (1996). The genus Nocardiopsis represents a phylogenetically coherent taxon and a distinct actinomycete lineage: proposal of Nocardiopsaceae fam. nov. Int J Syst Bacteriol 46, 10881092.

Sawyer, C. N. \& Ryckman, D. W. (1957). Anionic synthetic detergents and water supply problems. J Am Water Works Assoc 49, 480490.

Schleheck, D., Dong, W., Denger, K., Heinzle, E. \& Cook, A. M. (2000). An $\alpha$ proteobacterium converts linear alkylbenzenesulfonate (LAS) surfactants into sulfophenylcarboxylates, and linear alkyldi phenyletherdisulfonate surfactants into sulfodiphenylethercarboxy lates. Appl Environ Microbiol 66, 19111916.

Schleheck, D., Lechner, M., Schönenberger, R., Suter, M. J.-F. \& Cook, A. M. (2003). Desulfonation and degradation of sulfodiphe nylethercarboxylates from linear alkyldiphenyletherdisulfonate sur factants. Appl Environ Microbiol 69, 938944.

Schleheck, D., Knepper, T. P., Fischer, K. \& Cook, A. M. (2004). Mineralization of individual congeners of linear alkylbenzenesulfo nate (LAS) by defined pairs of heterotrophic bacteria. Appl Environ Microbiol 70, 40534063.
Schulz, S., Dong, W., Groth, U. \& Cook, A. M. (2000). Enantiomeric degradation of 2 (4 sulfophenyl)butyrate via 4 sulfocatechol in Delftia acidovorans SPB1. Appl Environ Microbiol 66, 19051910 .

Sittig, M. \& Hirsch, P. (1992). Chemotaxonomic investigation of budding and/or hyphal bacteria. Syst Appl Microbiol 15, 209222.

Sörbo, B. (1987). Sulfate: turbidimetric and nephelometric methods. Methods Enzymol 143, 36.

Strömpl, C., Tindall, B. J., Jarvis, G. N., Lünsdorf, H., Moore, E. R. B. \& Hippe, H. (1999). A re evaluation of the taxonomy of the genus Anaerovibrio, with the reclassification of Anaerovibrio glycerini as Anaerosinus glycerini gen. nov., comb. nov., and Anaerovibrio burkinabensis as Anaeroarcus burkinensis gen. nov., comb. nov. Int J Syst Bacteriol 49, 18611872.

Tindall, B. J. (1990a). A comparative study of the lipid composition of Halobacterium saccharovorum from various sources. Syst Appl Microbiol 13, 128130.

Tindall, B. J. (1990b). Lipid composition of Halobacterium lacusprofundi. FEMS Microbiol Lett 66, 199202.

Urakami, T. \& Komagata, K. (1987). Characterization and identification of methanol utilizing Hyphomicrobium strains and a comparison with species of Hyphomonas and Rhodomicrobium. J Gen Appl Microbiol 33, 521542.

van Ginkel, C. G., van Dijk, J. B. \& Kroon, A. G. (1992). Metabolism of hexadecyltrimethylammonium chloride by Pseudomonas strain B1. Appl Environ Microbiol 58, 30833087.

Ziemke, F., Höfle, M. G., Lalucat, J. \& Rosselló-Mora, R. (1998). Reclassification of Shewanella putrefaciens Owen's genomic group II as Shewanella baltica sp. nov. Int J Syst Bacteriol 48, 179186. 